\title{
Cryogenic-electron Microscopy for Battery Materials
}

\author{
Yuzhang $\mathrm{Li}^{1}$, Robert Sinclair ${ }^{2}$ and $\mathrm{Yi} \mathrm{Cui}^{2}$ \\ ${ }^{1}$ University of California, Los Angeles, Mountain View, California, United States, ${ }^{2}$ Stanford University, \\ Stanford, California, United States
}

Cryo-electron microscopy (cryo-EM) received the 2017 Nobel Prize in Chemistry for its ability to elucidate the nanostructure of biomolecules in their native state, revolutionizing the field of structural biology. Here, we pioneer an approach to utilize this powerful technique to enable new discoveries for batteries and show that cryo-EM can potentially have a similar impact in materials research [1]. Whereas conventional transmission electron microscopy (TEM) studies are unable to preserve the native state of chemically-reactive and beam-sensitive battery materials (e.g. Li metal) after operation, such materials remain pristine at cryogenic conditions. It is then possible to atomically resolve individual Li metal atoms and their interface with the solid electrolyte interphase (SEI). We observe that dendrites in carbonatebased electrolytes grow along the $\langle 111\rangle$ (preferred), $\langle 110\rangle$, or $\langle 211\rangle$ directions as faceted, singlecrystalline nanowires. These growth directions can change at kinks with no observable crystallographic defect. Furthermore, we reveal distinct SEI nanostructures formed in different electrolytes that explain why certain additives lead to better performance [2]. Following this initial work, we used cryo-EM to further study the SEI, a critical battery interface that forms on all battery electrodes whose exact mechanistic function is poorly understood at the atomic scale. It has been widely believed that the SEI chemistry is the major factor in regulating performance. In our present work using cryo-electron microscopy (cryo-EM), we show for the first time that it is instead the SEI nanostructure that ultimately dictates battery performance [3]. This surprising yet consistent finding provides an entirely new direction to explore for engineering the SEI nanostructure in battery materials. To engineer the appropriate SEI nanostructures, a detailed understanding of how the nanostructure initially forms and evolves is necessary. In this study [4], we track the voltage-dependent, stepwise evolution of the nanostructure and impedance of the SEI on $\mathrm{CuO}$ nanowires using cryo-EM and electrochemical impedance spectroscopy (EIS). In carbonate electrolyte, the SEI forms at $1.0 \mathrm{~V}$ vs $\mathrm{Li} / \mathrm{Li}+$ as a $3 \mathrm{~nm}$ thick amorphous SEI and grows to $4 \mathrm{~nm}$ at $0.5 \mathrm{~V}$; as the potential approaches $0.0 \mathrm{~V}$ vs $\mathrm{Li} / \mathrm{Li}+$, the $\mathrm{SEI}$ on the $\mathrm{CuO}$ nanowires forms an $8 \mathrm{~nm}$ thick inverted multilayered nanostructure in ethylene carbonate/diethyl carbonate (EC/DEC) electrolyte with 10 vol \% fluoroethylene carbonate (FEC) and a mosaic nanostructure in EC/DEC electrolyte. Upon Li deposition, the total SEI thickness grows to $16 \mathrm{~nm}$, and significant growth of the inner amorphous layer takes place in the inverted multilayered nanostructure, indicating that electrolyte permeates the SEI. Using a refined EIS methodology, we isolate the SEI impedance on $\mathrm{Cu}$ and find that the SEI nanostructure directly correlates to macroscopic Li-ion transport through the SEI. The inverted layered nanostructure decreases the interfacial impedance upon formation, whereas the mosaic nanostructure continually increases the interfacial impedance during growth. These structural and electrochemical findings illustrate a more complete portrait of SEI formation and guide further improvements in engineered SEI. With cryoEM, we open up exciting new opportunities for scientific discovery, which will be critical for providing fundamental insight to battery materials design [5].

\section{References}

1. Li, Y., Li, Y. \& Cui, Y. Catalyst: How Cryo-EM Shapes the Development of Next-Generation Batteries. Chem 4, 2250-2252 (2018).

2. Li, Y. et al. Atomic structure of sensitive battery materials and interfaces revealed by cryo-electron microscopy. Science 358, 506-510 (2017). 
3. Li, Y. et al. Correlating structure and function of battery interphases at atomic resolution using cryoelectron microscopy. Joule 2, 2167-2177 (2018).

4. Huang, W. et al. Nanostructural and Electrochemical Evolution of the Solid-Electrolyte Interphase on $\mathrm{CuO}$ Nanowires Revealed by Cryogenic-Electron Microscopy and Impedance Spectroscopy. ACS Nano 13, 737-744 (2018).

5. The authors acknowledge funding from Battery500 consortium programs. 